\title{
Evaluation of an Electronically Switched Directional Antenna for Real-world Low-power Wireless Networks
}

\author{
Erik Öström, Luca Mottola, Thiemo Voigt \\ Swedish Institute of Computer Science (SICS), Kista, Sweden
}

\begin{abstract}
We present the real-world evaluation of SPIDA, an electronically switched directional antenna. Compared to most existing work in the field, SPIDA is practical as well as inexpensive. We interface SPIDA with an off-the-shelf sensor node which provides us with a fully working real-world prototype. We assess the performance of our prototype by comparing the behavior of SPIDA against traditional omni-directional antennas. Our results demonstrate that the SPIDA prototype concentrates the radiated power only in given directions, thus enabling increased communication range at no additional energy cost. In addition, compared to the other antennas we consider, we observe more stable link performance and better correspondence between the link performance and common link quality estimators.
\end{abstract}

\section{Introduction}

The use of external antennas is a common design choice in many deployments of lowpower wireless networks [13]. Indeed, an external antenna often features higher gains compared to the antennas found aboard mainstream devices, enabling increased reliability in communication at no additional energy cost. To implement such design, researchers and domain-experts have hitherto borrowed the required technology from WiFi networks $[10,22]$. This holds both w.r.t. scenarios requiring omni-directional communication [22], and where the application at hand allows directional communication [10]. Although this implementation choice already enables improved performance, it is still sub-optimal in many respects, e.g., w.r.t. the significant size of the resulting devices, which complicates their installation. Unfortunately, as illustrated in Section 2, currently there are no practical solutions to address these issues, particularly in scenarios where some form of directional communication would be applicable.

To address this challenge, Nilsson designed SPIDA [11], an electronically switched directional antenna, shown in Figure 1. The SPIDA antenna is intended primarily for real-world low-power wireless networking, targeting scenarios that benefit from directional communication and sensor node localisation. We build a version of SPIDA that interfaces to a commercial sensor node—-the popular TMote Sky platform [12]—and design and implement the software drivers necessary to dynamically control the direction of maximum gain. Section 3 describes the hardware/software integration of SPIDA with the sensor network platform.

We evaluate the performance of our SPIDA prototype in a real-world setting, as described in Section 4. We compare the SPIDA behavior against two omni-directional 


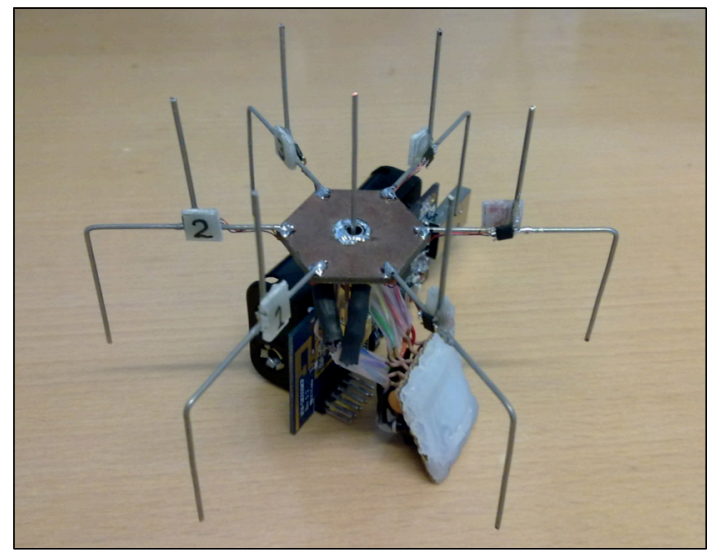

Fig. 1. SPIDA prototype, connected to a TMote Sky node.

antennas: an on-board micro-strip antenna and an external whip antenna for WiFi networks. We study the packet delivery rate and link quality using various network layouts, to assess communication ranges and directionality. To assess the dynamic abilities of SPIDA, we also run experiments by changing at run-time the direction of maximum gain. The results demonstrate that our SPIDA prototype behaves according to the intended design, and provides significant improvements in all metrics compared to the other antennas we consider.

The availability of a practical, inexpensive solution for dynamically controllable directional communication in low-power wireless networks raises interesting research questions and opens up a wealth of opportunities. We elaborate on this in Section 5, pointing to the network-level mechanisms that may leverage such antenna technology, and illustrating the expected performance gains.

We end the paper in Section 6 with brief concluding remarks.

\section{Related Work}

Nilsson identifies three candidate classes of directional antennas for low-power networks [11]: the adcock-pair antenna, the pseudo-doppler antenna, and the electronically switched parasitic element antenna. As described in Section 3, the SPIDA is an example of the latter class. At present, we could not find descriptions of other prototypes in any of these classes in the literature, let apart real-world experimental studies like ours.

The work closest to ours is that by Giorgietti et al. [8], who describe a prototype of four-beam patch antenna integrated with TMote Sky nodes, and related real-world experimental results. The direction of maximum gain is software-controlled, as in our SPIDA prototype. The size of the antenna, however, is much bigger than SPIDA. Giorgetti et al. leverage the experimental data to define analytical models for simulations. A similar activity using SPIDA is underway.

As already mentioned, antennas with fixed directions of maximum gain are employed in real-world applications [10,22], but also as deployment tools. For instance, 


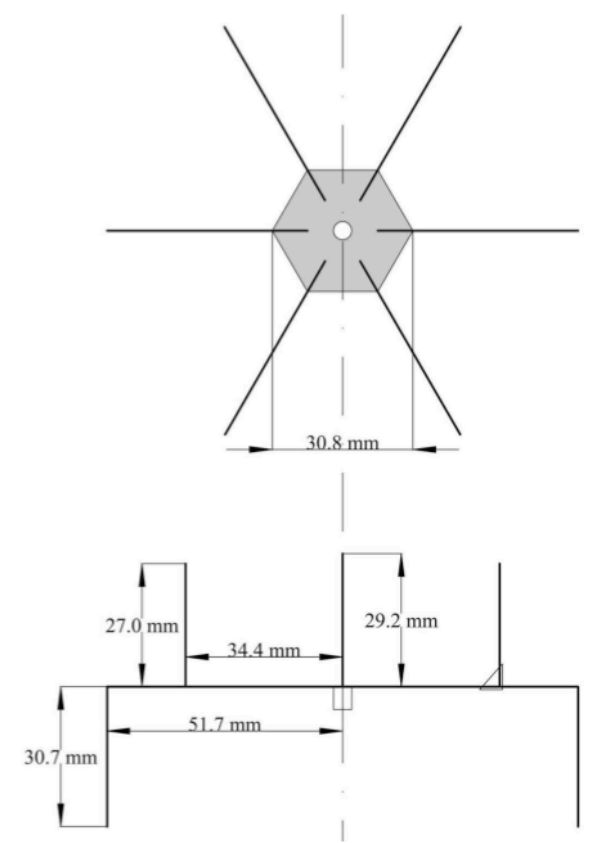

Fig. 2. SPIDA schematics without control electronics [11].

Saukh et al. [14] use "cantennas"- -simple cylinder-shaped directional antennas-for node localisation and selective communication to a group of nodes.

Despite the lack of real-world prototypes of dynamically controllable directional antennas, the benefits they provide motivated research efforts at both MAC and routing layer $[4,5,7,15,21]$, in low-power as well as mobile wireless networks. Most times, these leverage simulations or analytical studies based on abstract models of dynamically controllable directional antennas. Therefore, their behavior tends to be fairly idealized.

Advocating a top-down approach, some works provide guidelines for the design of dynamically controllable directional antennas based on the requirements imposed by higher-layer protocols $[19,23]$. On the contrary, our research activity around the SPIDA antenna leverages a bottom-up approach, starting from a practical real-world antenna prototype, and then aiming at designing networking mechanisms leveraging its features, as discussed in Section 5.

\section{Hardware/Software Design}

In this section we describe the SPIDA hardware and the related control software.

\subsection{Hardware}

The SPIDA antenna, developed at SICS by Nilsson [11], operates in the $2.4 \mathrm{GHz}$ ISM band. SPIDA is a switched parasitic element antenna [18], i.e., it consists of a central active element surrounded by "parasitic" elements, as shown in Figure 2. The former is a conventional quarter-wavelength whip antenna. The parasitic elements can be 


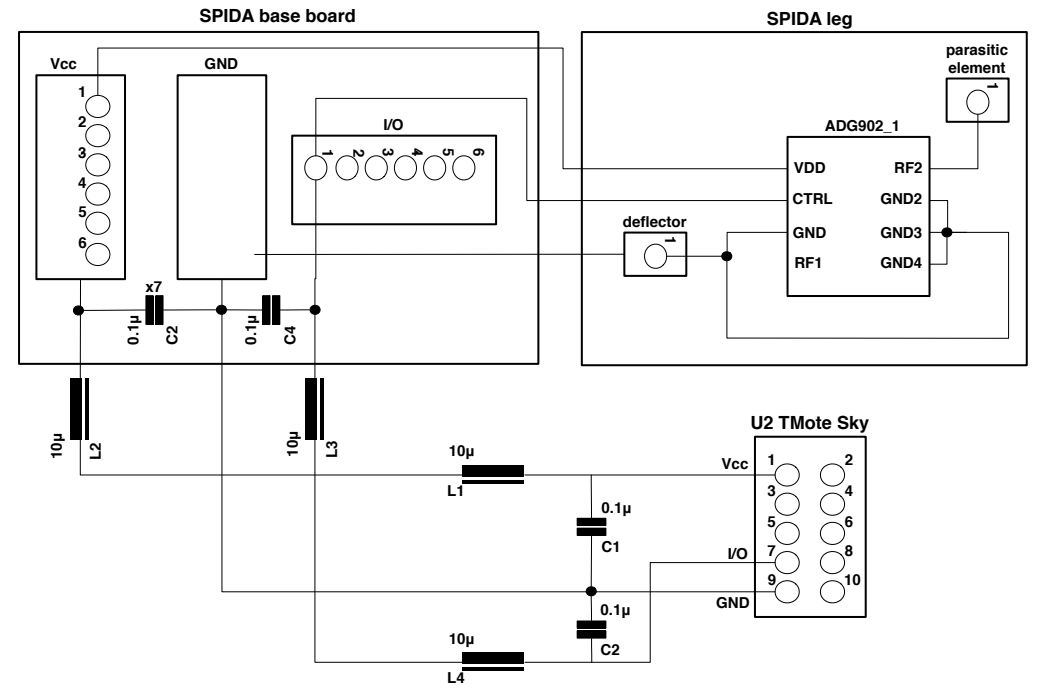

Fig. 3. SPIDA control electronics for a single parasitic element.

switched between ground and isolation. When grounded, they work as reflectors of radiated power, and when isolated they work as directors of radiated power. The SPIDA is equipped with six parasitic elements, yielding six possible "switches" to control the direction of transmission.

A distinguishing feature is the SPIDA's smoothly varying radiation pattern. The antenna gain is designed to vary as an offset circle from approximately $7 \mathrm{~dB}$ to $-4 \mathrm{~dB}$ in the horizontal plane, with the highest gain in the direction of the isolated parasitic elements. Although one may desire more selective transmission patterns, this choice simplifies the construction and use of the device, as we discuss in Section 5. In principle, such antenna behavior is obtained without any significant side lobes even when using simplistic on-off control [11]. The antenna is straightforward to manufacture, and its most expensive part is the SMA connector costing about 5 ECU in single quantities.

The circuitry to control the parasitic elements aims at reducing interference and suppressing noise from the sensor node digital circuitry. The schematics to control an individual parasitic element is shown in Figure 3. The available I/O lines on the TMote Sky are used to control the parasitic elements, using two LC filters for each I/O line to prevent noise from entering the RF section. Each parasitic element is controlled by an ADG902 SPST RF solid state switch. The control circuit is soldered onto a strip-board with an attached 10-pin IDC connector that fits onto the TMote Sky expansion pins.

\subsection{Software}

We design and implement the software drivers necessary to control the six parasitic elements aboard the SPIDA, targeting the Contiki operating system [6]. The API provided to programmers is simple, as shown in Figure 4. The first function initializes the driver. The following two functions are used to isolate or ground specific parasitic elements on the SPIDA, enabling individual fine-grained control. Nevertheless, we expect the 


\begin{tabular}{|l|c|l|}
\hline Function & Input & Description \\
\hline spida_init() & N/A & Initialize the driver. \\
\hline spida_activate (int) & $1-6$ & Isolate one of the six individual parasitic elements. \\
\hline spida_deactivate(int) & $1-6$ & Ground one of the six individual parasitic elements. \\
\hline spida_configure(int) & $0-6$ & $\begin{array}{l}\text { Configure all parasitic elements at once to set a specific direction of maximum gain. } \\
\text { (0 causes the SPIDA to behave as an omni-directional antenna). }\end{array}$ \\
\hline
\end{tabular}

Fig. 4. SPIDA driver API.

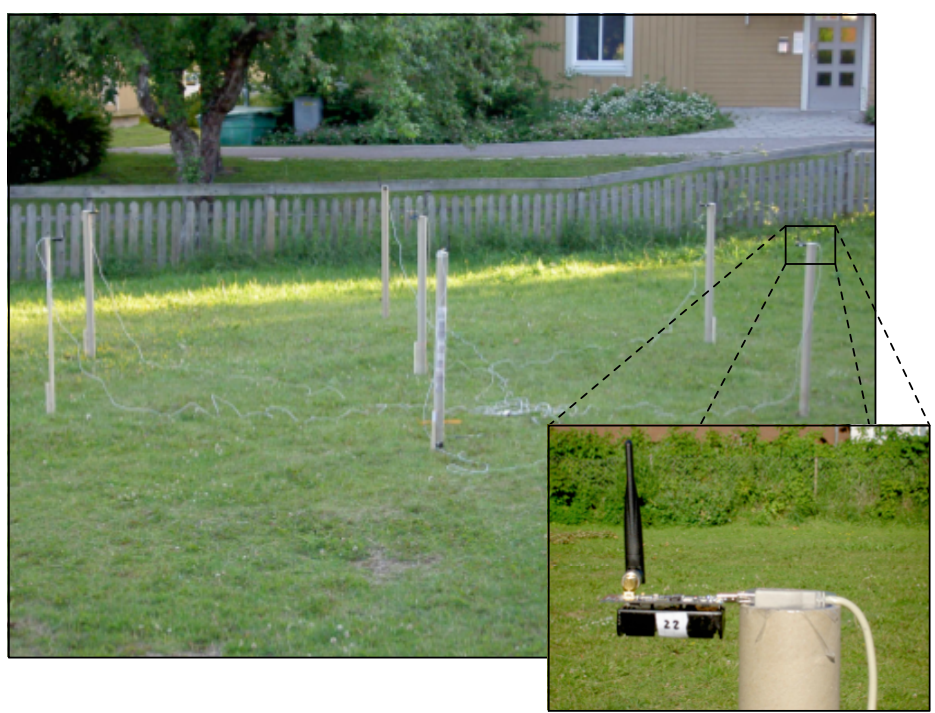

Fig. 5. Test environment and antenna orientation on probe nodes.

common use of the SPIDA to involve only one isolated element at a time, to direct the transmission in a specific direction. The last function in Figure 4 configures all parasitic elements at once to set a specific direction of maximum gain. Giving 0 as input makes the SPIDA isolate all parasitic elements, corresponding to omni-directional behavior. For instance, this may be useful for neighbor discovery.

\section{Real-world Evaluation}

We present the real-world evaluation we perform with our SPIDA prototype. Our objective is to investigate the SPIDA performance at the physical layer compared to the TMote Sky embedded microstrip antenna [20] and an external whip antenna for WiFi networks. The latter is connected to the node through a standard SMA connector and features a nominal gain of $2 \mathrm{~dB}$.

\subsection{General Setting}

We deploy the nodes in an open grass field, shown in Figure 5. The location we choose has no interference coming from other networks working in the ISM band. We 
verify this condition by taking periodic noise floor measurements during the experiments, also with the TMote Sky's CC2420 radio chip. We install the nodes atop $1 \mathrm{~m}$ tall cardboard pillars to avoid signal reflections from the ground [3], and power them through the USB connector to factor out the influence of the battery discharge. All antennas we consider are oriented with the radiating element orthogonal to the ground, as shown at the bottom right of Figure 5. We carry out all experiments in comparable conditions of humidity and temperature. We check these conditions during the experiments by periodically querying the TMote Sky's integrated SHT11 sensor.

The various scenarios we investigate differ in the network layout, as described next. In every case, however, one node transmits using different antennas, while the others operate as passive probes, logging the received packets. The probes employ the external whip antenna shown in Figure 5. The SPIDA is always configured with only one parasitic element isolated: the configuration that yields the highest degree of directional transmission. For each experiment, the transmitter sends 1000 packets with an inter-packet interval of $500 \mathrm{~ms}$. We use the lowest power setting, which enables easier logistics. The experiment code is implemented on top of the Contiki [6] operating system, and uses channel 26 for the transmissions.

As performance metrics, we consider averages over all probe nodes of the following figures: $i)$ the packet delivery rate $(P D R)$, defined as the average number of packets received at a probe over those sent by the transmitter, ii) the received signal strength $(R S S I)$, and iii) the link quality indicator $(L Q I)$. We obtain the two latter for every received packet directly from the $\mathrm{CC} 2420$ radio chip. Because of this, the charts for $R S S I$ and $L Q I$ do not show regions where no packets were received. The results described next are averages over at least 5 repetitions of every experiment.

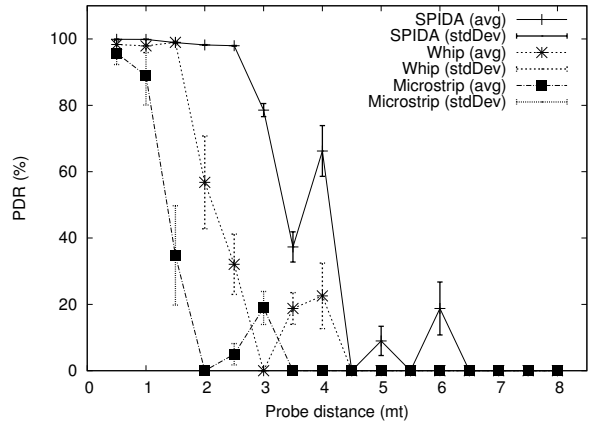

(a) PDR

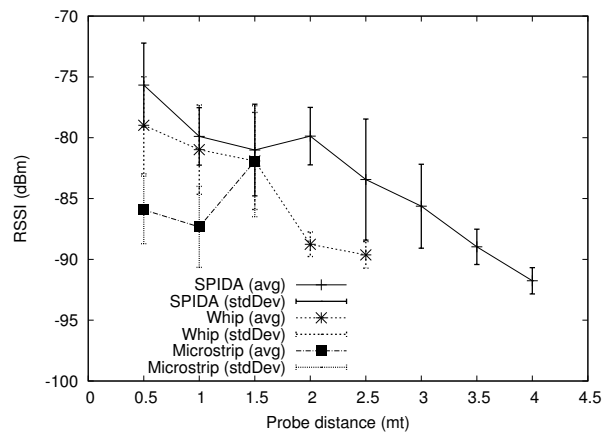

(b) $R S S I$.

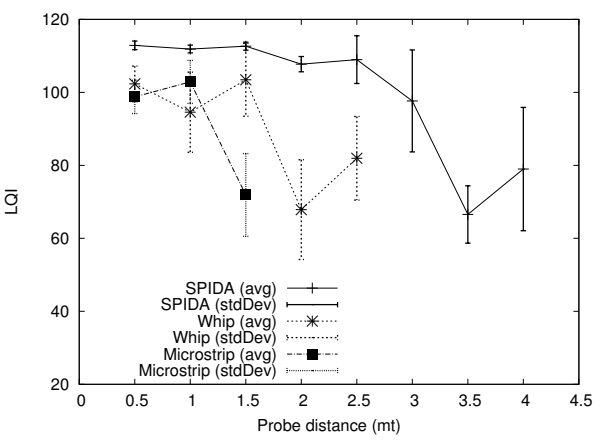

(c) $L Q I$.

Fig. 6. The SPIDA antenna extends the radio range and enjoys better correspondence between $L Q I$ and $P D R$ compared to the other antennas. 


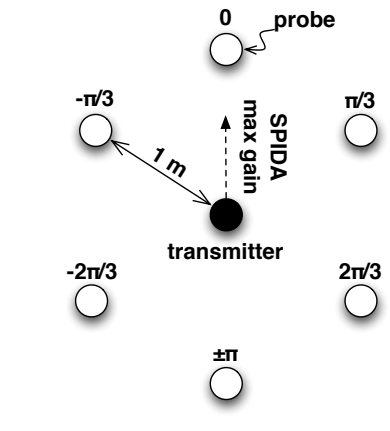

(a) Coarse-grained experiments.

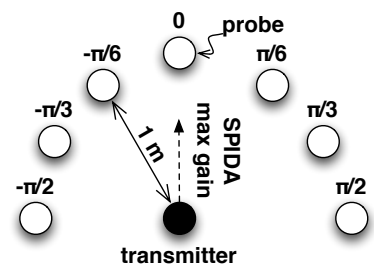

(b) Fine-grained experiments.

Fig. 7. Network layout for directional experiments.

\subsection{Network Layouts and Results}

We describe next the specific network layout in every experiment and report on the corresponding results.

Range experiments. We compare the communication range of the SPIDA antenna against the other antennas we consider. To do so, we use only one probe node, placed at varying distances from the transmitter. In the first round of these experiments, the SPIDA has the isolated parasitic element pointing towards the probe.

Figure 6 illustrates the results. As shown in Figure 6(a), in the direction of maximum gain the SPIDA reaches much farther than the other two antennas. Using the SPIDA, the "connected" region [24] with $P D R$ above $90 \%$ is about twice that of the whip antenna, and four times the case of the microstrip one. This is a key metric, as it indicates the portion of space characterized by reliable communication. The SPIDA also extends the "grey area" [24], characterized by highly varying performance and no predictable behavior. This is also an effect of the extended communication range.

The result above is reflected in the trends for RSSI and $L Q I$, shown in Figure 6(b) and 6(c). Moreover, within the connected region the SPIDA shows better correspondence between $L Q I$ and $P D R$ than the other antennas. Thus, with comparable link performance in $P D R$, link quality estimators based on $L Q I$ [17] are likely to perform better with the SPIDA.

We also repeat the experiment with the isolated parasitic element of the SPIDA pointing in the direction opposite to the probe. Using this setting, the probe always receives less than 10 packets at $0.5 \mathrm{~m}$ from the transmitter, and then nothing beyond $1 \mathrm{~m}$. This is a first evidence that the SPIDA does direct the transmitted power in a given direction. We investigate these aspects further in the following experiments.

Coarse-grained directional experiments. We aim at a first, coarse grained characterization of the spatial characteristics of SPIDA transmissions compared to the other two antennas. To this end, we place the transmitter in the center of a circle of six probe nodes, as shown in Figure 7(a). Based on the results of the range experiments, we place the probes at $1 \mathrm{~m}$ from the transmitter, corresponding to the connected region for all antennas. We place the probes with the TMote Sky's USB connector pointing towards the transmitter. When using the SPIDA, every probe is aligned with a parasitic element. 
We show the results in Figure 8. As depicted in Figure 8(a), the SPIDA achieves about $100 \%$ PDR only along the direction of maximum gain, corresponding to the isolated parasitic element. We also observe that the transmission pattern forms a lobe large enough to cover the probes at $\pm \frac{\pi}{3}$ as well, which still receive a significant number of packets. Nevertheless, the probes at $\pm \frac{2 \pi}{3}$ and $\pm \pi$ receive no packets at all. This behavior largely corresponds to the simulation results reported earlier [11]. Thus, despite its simplicity, the electronics we built have very little influence on the antenna performance. As expected, the whip antenna shows an almost perfect omni-directional behavior. On the other hand, the microstrip antenna suffers from the co-location with the node base board, showing a drop in $P D R$ around $\frac{\pi}{3}$. Such behavior is consistent with previous findings [20].

Figure $8(\mathrm{~b})$ and $8(\mathrm{c})$ illustrate the trends in $R S S I$ and $L Q I$, respectively. The SPIDA shows a maximum in RSSI along the direction of maximum gain, confirming the correct functioning of the electronics to control the parasitic elements. The same observation applies to the trends in $L Q I$. Both points of maxima also show less variability in the results than at $\pm \frac{\pi}{3}$, indicating a more stable link performance in the direction of maximum gain. On the other hand, both the whip antenna and the microstrip antenna show no clear trend in RSSI or $L Q I$. When using omni-directional antennas, these metrics are known not to show a clear correspondence with $P D R$ in most cases [16].

Fine-grained directional experiments. We investigate the transmission pattern of the SPIDA antenna at a finer grain around the direction of maximum gain. We deploy seven probes in a half-circle configuration, as in Figure 7(b). The other parameters are as in the previous coarse-grained experiments.

The results we obtain this time are shown in Figure 9. Figure 9(a) demonstrates the smoothly varying radiation pattern of the SPIDA. The PDR gradually decreases between 0 degrees-which is aligned with the isolated parasitic element-and $\pm \frac{\pi}{3}$, 
until it drops to zero at $\pm \frac{\pi}{2}$. Again the whip antenna behaves in an omni-directional manner, whereas the microstrip shows a larger drop around $\frac{\pi}{3}$, due to the higher spatial resolution of these experiments.

The trends in $R S S I$ and $L Q I$, shown in Figure 9(b) and 9(c), confirm our observations. With the SPIDA, the decrease in both metrics is gradual around the direction of maximum gain, and the variability is reduced along this direction compared to both the other two antennas and the other directions with the SPIDA.

Dynamic experiments. We also test the SPIDA's ability to change the direction of maximum gain at run-time. We use again the network layout in Figure 7(a). However, this time we program the transmitter to switch the isolated parasitic element after every packet, moving the direction of maximum gain clockwise in the horizontal plane. We repeat this experiment 10 times.

Figure 10 illustrates the trends in the metrics we consider as a function of a given probe, against the current direction of maximum gain. All results are remarkably consistent no matter which probe we examine. For instance, Figure 10(a) shows that all probes observe the same behavior in $P D R$ as the direction of maximum gain changes, with the only difference of a variable offset due to a probe's relative displacement. It also appears that the SPIDA slightly favors the $P D R$ at the probe to the left of the direction of maximum gain. This behavior is presumably due to some little imperfections in the construction process, which can be easily rectified.

The same observations apply to the results in $R S S I$ and $L Q I$, depicted in Figure $10(\mathrm{~b})$ and $10(\mathrm{c})$. Both show a peak at the probe aligned with the current direction of maximum gain, and a reasonably symmetric decrease of the same metric at the two adjacent probes. The variability of both $R S S I$ and $L Q I$ (not shown in the charts) is comparable to the other experiments.

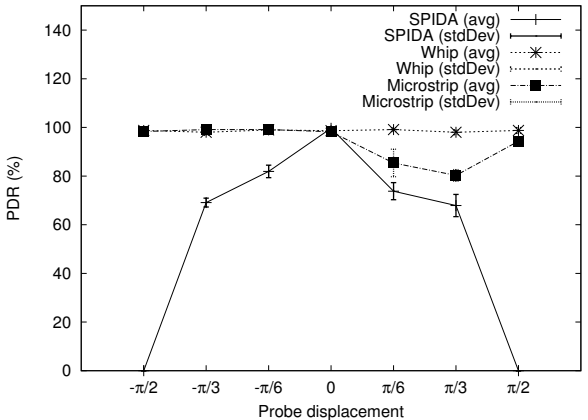

(a) $P D R$

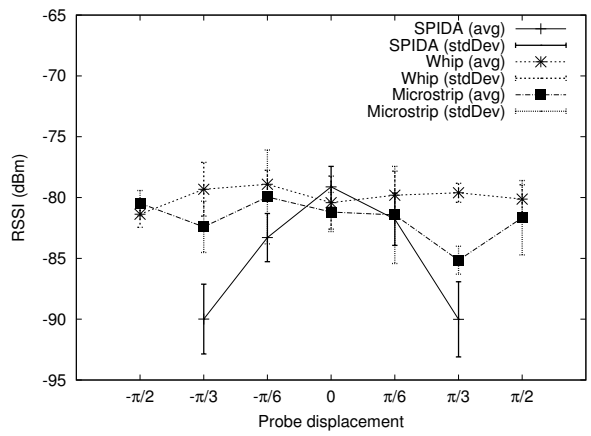

(b) $R S S I$.

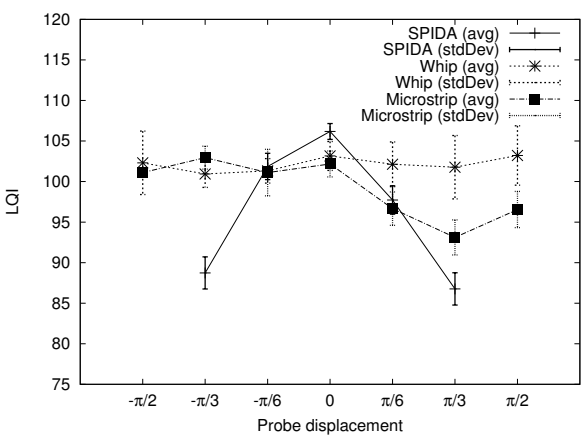

(c) $L Q I$.

Fig. 9. The fine-grained directional experiments again demonstrate SPIDA's directionality w.r.t. all metrics. 


\section{Outlook}

From a networking perspective, the availability of a SPIDA-like prototype raises interesting research questions and opens up several opportunities.

For instance, we believe that there may be significant advantages by leveraging a SPIDA-like antenna are at the routing layer. Consider the classical multi-hop, convergecast scenario using tree-shaped routing topologies. By using directed transmissions towards the parent node, one may diminish the probability of collisions due to simultaneous transmissions along parallel paths. This would provide greater reliability and reduce energy consumption by decreasing the number of necessary retransmissions.

However, achieving this functionality is not necessarily trivial. For instance, one may devise directionality-aware parent selection mechanisms, or re-use existing schemes and simply use directional transmissions when sending to the parent. In the latter case, the increase in communication range, which we also observed with the SPIDA in Section 4, may allow transmissions to reach nonparent nodes that are however closer to the sink. Significant trade-offs are involved in devising similar functionality, e.g., complexity vs. communication overhead, which deserve careful investigation.

Another example is related to the use of dynamically controllable directional antennas in TDMA-like MAC protocols. Doing so may enable spatial diversity in addition to time diversity. In this context, the few existing solutions tend to be very complex [21]. However, the SPIDA's radiation pattern, characterized by a simple offset circle, may greatly simplify the problem at the cost of slightly increased contention on the wireless medium. Here again, the trade-off between the degree of directional communication and the simplifications in the MAC operation shall be analyzed thoroughly.

Even staple networking mechanisms such as neighbor discovery may benefit form the use of dynamically controllable directional antennas. How to leverage this function- 
ality, however, is an open question. If the antenna also provides omni-directional behavior, as in the case of SPIDA, one may re-use existing mechanisms. However, when the antenna turns to directional mode, the increased transmission range may reach nodes that were previously not recognized as neighbors. This would impact the operation of MAC protocols, as topology information would suddenly become inconsistent. Topology control schemes [9] may decrease the transmission power to maintain the same neighboring relations when the antenna is operating in directional mode. However, this would partly defeat the increased reliability obtained with directional transmissions.

On the other hand, one may use directional mode for neighbor discovery as well, rapidly sweeping all possible directions. However, by doing so, the link quality to different neighbors would be sampled at slightly different times, which might affect the operation of higher-level mechanisms, especially multi-hop routing protocols [1]. Most existing works in this area assume a priori knowledge on node positions. Even though directional antennas like the SPIDA are used for localization based on angle-of-arrival information [2], we do need much better integration of these functionality.

\section{Conclusion}

In this paper we reported on real-world experiments with SPIDA, an electronically switched directional antenna for low-power wireless networks. We showed that SPIDA concentrates the radiated power only in given directions. Based on a comparison with the on-board micro-strip antenna of the TMote Sky node and an external whip antenna, we observed increased communication range, improved link stability, and better correspondence between link performance and common link quality estimators. As we illustrated, this opens up several opportunities for improved network-level mechanisms that leverage the characteristics of SPIDA-like antennas.

Acknowledgements. We thank Martin Nilsson (SICS) who designed the SPIDA antenna and advised us on interfacing SPIDA to a TMote Sky sensor node. This work was supported by VINNOVA, the Uppsala VINN Excellence Center for Wireless Sensor Networks WISENET, also partly funded by VINNOVA, and CONET, the Cooperating Objects Network of Excellence, under EU-FP7 contract number FP7-2007-2-224053.

\section{References}

1. J. Al-Karaki and A. E. Kamal. Routing techiniques in wireless sensor networks: A survey. IEEE Wireless Communications, 11(6), 2004.

2. I. Amundson, J. Sallai, X. Koutsoukos, and A. Ledeczi. Radio interferometric angle of arrival estimation. In Proc. of the $7^{\text {th }}$ European Conf. on Wireless Sensor Networks (EWSN), 2010.

3. G. Anastasi, E. Borgia, M. Conti, E. Gregori, and A. Passarella. Understanding the real behavior of Mote and 802.11 ad hoc networks: An experimental approach. Elsevier Pervasive and Mobile Computing Journal, 1(2):237-256, 2005.

4. O. Bazan and M. Jaseemuddin. On the design of opportunistic MAC protocols for multi-hop wireless networks with beamforming antennas. IEEE Transactions on Mobile Computing, 99(PrePrints), 2010. 
5. J. Cho, J. Lee, T. Kwon, and Y. Choi. Directional antenna at sink (DAaS) to prolong network lifetime in wireless sensor networks. In Proc. of the European Wireless Conf. - Enabling Technologies for Wireless Multimedia Communications, 2006.

6. A. Dunkels, B. Grönvall, and T. Voigt. Contiki - A lightweight and flexible operating system for tiny networked sensors. In Proc. of $1^{\text {st }}$ Wkshp. on Embedded Networked Sensors, 2004.

7. J. Dunlop and J. Cortes. Co-design of efficient contention mac with directional antennas in wireless sensor networks. In Proc. of the International Wireless Communications and Mobile Computing Conference, 2008.

8. G. Giorgetti, A. Cidronali, S. Gupta, and G. Manes. Exploiting low-cost directional antennas in 2.4 GHz IEEE 802.15.4 wireless sensor networks. In Proc. of the European Conf. on Wireless Technologies, 2007.

9. G. Hackmann, O. Chipara, and C. Lu. Robust topology control for indoor wireless sensor networks. In Proc. of the $6^{\text {th }}$ Int. Conf. on Embedded Networked Sensor Systems (SenSys), 2008.

10. S. Kim, S. Pakzad, D. Culler, J. Demmel, G. Fenves, S. Glaser, and M. Turon. Health monitoring of civil infrastructures using wireless sensor networks. In Proc. of the $6^{\text {th }}$ Int. Conf. on Information Processing in Sensor Networks (IPSN), 2007.

11. M. Nilsson. Directional antennas for wireless sensor networks. In Proc. of the $9^{\text {th }}$ Scandinavian Workshop on Wireless Adhoc Networks (Adhoc), 2009.

12. J. Polastre, R. Szewczyk, and D. Culler. Telos: Enabling ultra-low power wireless research. In Proc. of the $5^{\text {th }}$ Int. Conf. on Information Processing in Sensor Networks (IPSN), 2005.

13. B. Raman and K. Chebrolu. Censor networks: a critique of "sensor networks" from a systems perspective. SIGCOMM Comput. Commun. Rev., 38(3), 2008.

14. O. Saukh, R. Sauter, J. Meyer, and P. Marrón. Motefinder: A deployment tool for sensor networks. In Proc. of the Workshop on Real-World Wireless Sensor Networks (REALWSN), 2008.

15. E. Shihab, L. Cai, and J. Pan. A distributed, asynchronous directional-to-directional MAC protocol for wireless ad hoc networks. IEEE Trans. on Vehicular Technology, 58(9), 2009.

16. K. Srinivasan, P. Dutta, A. Tavakoli, and P. Levis. An empirical study of low power wireless. ACM Transactions on Sensor Networks, 2010. To appear.

17. K. Srinivasan and P. Levis. RSSI is under-appreciated. In Proc. of the $3^{\text {nd }}$ Int. Workshop on Embedded Networked Sensors (EmNets), 2006.

18. D. Thiel and S. Smith. Switched parasitic antennas for cellular communications. Artec House, London, 2002.

19. Q. Tian, S. Bandyopadhyay, and E. J. Coyle. The effect of directional antennas on spatiotemporal sampling in clustered sensor networks. J. of Internet Technology (JIT), 8(1), 2007.

20. TMote Sky Datasheet. www.snm.ethz.ch/pub/uploads/Projects/tmote_ sky_datasheet.pdf.

21. R. Vilzmann and C. Bettstetter. A survey on MAC protocols for ad hoc networks with directional antennas. EUNICE 2005: Networks and Applications Towards a Ubiquitously Connected World, 196(1), 2006.

22. G. Werner-Allen, K. Lorincz, J. Johnson, J. Lees, and M. Welsh. Fidelity and yield in a volcano monitoring sensor network. In Symp. on Operating Systems Design and Implementation (OSDI), 2006.

23. Y. Wu, L. Zhang, Y. Wu, and Z. Niu. Interest dissemination with directional antennas for wireless sensor networks with mobile sinks. In Proc. of the $6^{\text {th }}$ Int. Conf. on Embedded Networked Sensor Systems (SenSys), 2006.

24. J. Zhao and R. Govindan. Understanding packet delivery performance in dense wireless sensor networks. In Proc. of the $3^{\text {rd }}$ Int. Conf. on Embedded Networked Sensor Systems (SenSys), 2003. 